\title{
Tea Polyphenols as Natural Products for Potential Future Management of HIV Infection - An Overview
}

\author{
Hamid Reza Sodagari ${ }^{1}$, Roodabeh Bahramsoltani ${ }^{2}$, Mohammad Hosein Farzaei ${ }^{3}$, \\ Amir Hossein Abdolghaffari ${ }^{4}$, Nima Rezaei ${ }^{5}$ and Andrew W. Taylor-Robinson ${ }^{6 *}$ \\ ${ }^{1}$ Young Researchers \& Elite Club, Karaj Branch, Islamic Azad University, \\ Karaj, Iran \\ 2 Faculty of Pharmacy, Tehran University of Medical Sciences, Tehran, Iran \\ ${ }^{3}$ Medical Biology Research Center, Kermanshah University of Medical Sciences, \\ Kermanshah, Iran \\ ${ }^{4}$ Pharmacology \& Applied Medicine Department of Medicinal Plants Research Center, \\ Institute of Medicinal Plants, ACECR, Karaj, Iran \\ ${ }^{5}$ Research Center for Immunodeficiency, Children's Medical Center, \\ Tehran University of Medical Sciences, Tehran, Iran \\ ${ }^{6}$ School of Medical \& Applied Sciences, Central Queensland University, \\ Rockhampton, Australia
}

\begin{abstract}
Belonging to the Lentivirus genus of animal retroviruses, human immunodeficiency virus (HIV) is the etiological agent of acquired immunodeficiency syndrome (AIDS) which attacks cells of the immune system including $C D 4^{+} T$ lymphocytes, monocytes, macrophages and dendritic cells. A rapid progression to immunodeficiency and the higher transmissibility of HIV-1 compared to HIV-2 are hallmarks of the worldwide spread of AIDS. Conventional HIV treatments are limited by drug toxicity and by multi-drug resistance, caused by the high genetic variability of HIV. This has led researchers into new areas of drug discovery in search of novel therapeutic molecules. Accumulating evidence indicates that tea polyphenols possess a range of beneficial properties including anti-cancer, anti-inflammatory, anti-oxidative, neuro-protective, antibacterial, anti-fungal and anti-viral effects. The anti-HIV infection potential of tea polyphenols has been confirmed by several preclinical studies. This suggests that polyphenol-rich extracts of tea could be used as dietary supplements as part of a combined therapeutic regimen with conventional anti-HIV drugs. Phenolic structures may also be considered as backbones for the discovery of a new generation of anti-HIV remedies. This review provides a perspective on the antiHIV activity of tea polyphenols and their development as a possible source of future drugs for the therapy of HIV/AIDS.
\end{abstract}

Keywords: AIDS, Catechin, HIV, Polyphenol, Phytomedicine, Tea, Theaflavin

\footnotetext{
${ }^{*}$ Author for correspondence

Email: a.taylor-robinson@cqu.edu.au
} 


\section{Introduction}

\subsection{HIV Aetiology and Pathophysiology}

Human Immunodeficiency Virus (HIV) is a member of the Lentivirus genus, part of the Retroviridae family of enveloped, single-stranded, positive-sense RNA viruses that are characterized by replication in a host cell through the process of reverse transcription. HIV is the etiological agent of Acquired Immuno Deficiency Syndrome (AIDS) which infects cells of the human immune system including $\mathrm{CD}^{+} \mathrm{T}$ lymphocytes, monocytes, macrophages and dendritic cells ${ }^{1}$. As a zoonotic disease HIV/AIDS is thought to be derived from simian immunodeficiency viruses that infect West African primates ${ }^{2}$. Common chimpanzees (Pan troglodytes) and western lowland gorillas (Gorilla gorilla gorilla), and sooty mangabey monkeys (Cercocebus atys), are recognized as the original source of HIV1 and HIV-2, respectively. Of the two major types of HIV, such intrinsic features of HIV-1 as a greater rate of transmission than HIV-2 and driving a quicker loss of host immunocompetence have enabled it to become the principal cause of AIDS around the world ${ }^{2} . \mathrm{M}, \mathrm{N}$, $\mathrm{O}$ and $\mathrm{P}$ are the four identified groups of HIV-1 that are isolated from chimpanzees and gorillas. Of these, group $\mathrm{M}$, which comprises nine subtypes: A-D, F-H, J and $\mathrm{K}$, is known to be responsible for the global HIV pandemic. This group was transmitted to humans in West-Central Africa approximately 100 years ago ${ }^{3,4}$.

The globalization of HIV-1 may be due to the high mutation and recombination rates of the nine subtypes of group $\mathrm{M}$ caused by the Reverse Transcriptase (RT) enzyme 5 . The number of infections caused by recombinants is reported to have increased from $17 \%$ over the period $2000-07$ to at least $20 \%$ by $2011^{6}$. Drug injection with contaminated shared needles ${ }^{7}$, unprotected sexual contact, percutaneous blood exposure, mucous membrane exposure to contaminated blood or other body fluids, mother-to-child transmission and receipt of contaminated blood products are all identified as major routes of HIV transmission ${ }^{8}$. After gaining entry into the body via one of these routes, each virion attacks a $\mathrm{CD} 4^{+}$ T lymphocyte, its main target, with the assistance of chemokine co-receptors such as CCR5 or CXCR4. The virus genome is released into the host cell and viral RT converts viral RNA into double stranded DNA. This new viral DNA is concealed within the DNA of the host cell by another HIV enzyme named integrase. In this way, transcription of the host cell's DNA during cell division causes spread of the virus within the host's body ${ }^{9-11}$.

\subsection{HIV Epidemiology}

It is estimated that worldwide over 35 million people live with HIV ${ }^{12}$, of whom $69 \%$ are resident in sub-Saharan Africa where 1 in every 20 adults is HIV-positive ${ }^{13}$. As far back as $20096 \%$ of Kenyan adults (15-49 years) were infected with HIV-1, with a difference between urban $(10 \%)$ and rural areas $(5.6 \%)^{14}$. Furthermore, the Centers for Disease Control and Prevention estimate that currently approximately 50,000 people contract HIV each year in the USA ${ }^{15}$. Another report from 2015 indicated that in China 501,000 people had HIV/AIDS among whom 205,000 were living with AIDS $^{16}$.

\subsection{Current Treatment of AIDS Complications}

There are more than 25 Anti-retroviral (ARV) drugs licensed for HIV therapy which are divided into several groups including nucleoside RT inhibitors (zidovudine, didanosine, zalcitabine, stavudine, lamivudine, abacavir, emtricitabine), nucleotide RT inhibitor (tenofovir), non-nucleoside RT inhibitors (nevirapine, delavirdine, efavirenz and etravirine), protease inhibitors (saquinavir, ritonavir, indinavir, nelfinavir, amprenavir, lopinavir, atazanavir, fosamprenavir, tipranavir and darunavir), fusion inhibitor (enfuvirtide), co-receptor inhibitor (maraviroc) and integrase inhibitor (raltegravir) ${ }^{17}$. Several side effects including constipation, fever, liver disorders, muscular dystrophy, lipoatrophy, metabolic disorders, blood disorders, hypersensitivity reactions, peripheral neuropathy, hyperlactaemia, pancreatitis and mitochondrial toxicity ${ }^{1,18,19}$, as well as multi-drug resistance and toxicity caused by the high genetic variability of HIV have limited long term treatment with the aforementioned ARV drugs ${ }^{20}$. Thus, screening of alternative drugs such as plant metabolites used as natural products would appear necessary due to their therapeutic activities with lesser or no side effects. Against this background, the principal purpose of this review is to provide an overview of recent findings on anti-HIV activity of polyphenols extracted from different types of tea. 


\section{Health Benefits of Tea Polyphenols as Dietary Natural Products}

Recently, polyphenols have been recognized as extremely important plant-derived dietary compounds due to producing a vast range of effects considered to be beneficial to humans. This comprises antioxidant, antibacterial, antiviral, anti-allergic, anti-colitis, anti-inflammatory, anti-arthritic and anti-cancer activities ${ }^{21-25}$. Among several polyphenol sources in the human diet, tea is one of the most consumed beverages around the world as a result of the pleasant taste, attractive aroma and health-promoting effects. Depending on the degree of processing and fermentation, tea is divided into green tea, which is consumed mostly in South East Asian countries, black tea, which is commonly consumed in the Indian subcontinent and Western societies, and Pu-erh, white, yellow and oolong teas that are produced and drunk mainly in China ${ }^{26}$. There are many reports about consumption of tea worldwide. The UK Tea Council claims that British people drink 60.2 billion cups of tea per year ${ }^{27}$. Worldwide, over 4.52 million tons of tea were produced in $2014^{28}$. In addition to the abundant distribution of polyphenols in this plant species, tea is also a leading natural source of caffeine, a psychoactive alkaloid that acts as a stimulant and, if taken at a bioactive dose, can show beneficial effects on wellbeing ${ }^{29}$.

While used as a routine part of the human diet, tea has attracted scientific attention due mostly to its highly active antioxidant and anti-inflammatory polyphenolic compounds, especially catechins ${ }^{29}$. The European Food Safety Authority reported that each $100 \mathrm{ml}$ of green tea contains about $126 \mathrm{mg}$ of catechin derivatives $^{30}$. Several beneficial properties have been attributed to tea polyphenols, including anti-cancer ${ }^{31,32}$, anti-diabetic ${ }^{33}$, anti-oxidative ${ }^{34}$, cardioprotective ${ }^{35}$ and neuroprotective ${ }^{36}$ activities, as well as antibacterial ${ }^{37}$, antifungal $^{38}$ and antiviral ${ }^{39,40}$ functions. Considering the high content of antioxidants and anti-inflammatory components, including flavonoids, tea could be introduced as a suitable beverage for the prevention of chronic inflammatory and oxidative stress-related conditions ${ }^{41}$.

\section{Anti-HIV Activity of Tea Polyphenols}

\subsection{Green Tea Polyphenols}

Green tea originates in China and is prepared from leaves of the evergreen shrub Camellia sinensis that have undergone minimal oxidation during processing. Monomeric flavanols, recognized as catechins, are the most important polyphenols with several beneficial therapeutic properties such as prophylactic effects on atherosclerosis, anti-oxidative stress, antiulcer, anti-bacterial and anti-cancer activities ${ }^{42-47}$. Catechins constitute $30-40 \%$ of the dry weight of fresh green tea leaves ${ }^{48}$, including (-)-epicatechin (EC), (-)-epicatechin gallate (ECG), (-)-epigallocatechin (EGC) and (-)-epigallocatechin gallate (EGCG) as main catechin derivatives, and also (-)-catechin gallate (CG), (-)-gallocatechin (GC), (-)-gallocatechin gallate (GCG), (-)-epigallocatechin 3,5-digallate (EGCDG) and 2 ',2"-bis epigallocatechin digallate (BGCDG) as minor ingredients ${ }^{40}$. EGCG is the most frequent catechin derivative of green tea polyphenols ${ }^{49}$.

The immunoregulatory effects of green tea extracts have been reported ${ }^{50-53}$. A dose-dependent decrease in histamine production was noted for rodent peritoneal mast cells incubated with green tea polyphenol ${ }^{50}$. Further experiments by these authors showed that the altered histamine release was due to a green tea extract-mediated decrease in cAMP and calcium levels which leads to an $\mathrm{NF \kappa B}$ and p38 MAPK-dependent inhibition of the proinflammatory cytokines TNF- $\alpha$ and IL- $6^{50}$. Green tea components are reported to have immunoregulatory activity. EGCG was effective at suppressing peripheral blood mononuclear cell proliferation and IFN- $\gamma$ production $^{51}$. They can also change the profile of proinflammatory cytokines secreted by lymphocytes ${ }^{52}$. Neutrophils were modulated by green tea polyphenols through suppression of the TLR-4/NFkB p65 signal activation pathway ${ }^{53}$.

EGCG inhibits HIV-1 replication in several steps of the virus life cycle by interfering with RT and protease activity, blocking gp120-CD4 interaction via binding to CD4 and destroying virions ${ }^{54-58}$. Several investigations have demonstrated that green tea catechins, especially EGCG, possess anti-HIV activity. For instance, it was 
shown that EGCG inhibits the infectivity-enhancing property of Semen-derived Enhancer of Virus Infection (SEVI) at non-toxic concentrations, treatment with 0.4 $\mathrm{mM}$ of EGCG reducing the average rate of infection by $70.6 \%(\mathrm{p}<0.0001)^{59}$. SEVI and also semen-mediated enhancement of HIV-1 infection may be inhibited by EGCG without cellular toxicity. In addition, the formation of fibril is abrogated completely by treatment with high concentrations of EGCG (10 and $20 \mathrm{mM}$ EGCG) ${ }^{60}$. Furthermore, it has been reported that blockade of antibody and glycoprotein 120 through binding of EGCG to $\mathrm{CD}^{+} \mathrm{T}$ cell is another anti-HIV mechanism of this molecule ${ }^{54}$. Prevention of attachment of HIV-1glycoprotein 120 to $\mathrm{CD} 4$ has a key role in inhibition of HIV-1 infection by EGCG ${ }^{61}$. Also, inhibition of HIV-1 p24 antigen production across a broad spectrum of both HIV-1 clinical isolates and laboratory adapted subtypes (B, C, D and G) has been observed following application of EGCG at a concentration of $6 \mu \mathrm{mol} / \mathrm{L}$.

Inhibition of HIV-1 infection by EGCG is not a consequence of cytotoxicity, cell growth inhibition or apoptosis, indicating the specificity of the inhibitory potential of EGCG for HIV-1 infectivity ${ }^{62}$. Treatment of HIV-1 Tat transgenic mice with EGCG at a dose of $300 \mathrm{mg} / \mathrm{kg} /$ day caused a reduction in Glial Fibrillary Acidic Protein (GFAP) associated neuronal loss ${ }^{63}$. EGCG both mildly reduced activated microgliosis and enhanced neuron survival. Antiviral activity of EGCG occurs by interacting with several steps of the HIV-1 life cycle. A destructive effect on virions, post-adsorption entry and RT in acutely infected monocytoid cells were also observed at concentrations of EGCG greater than $1 \mu \mathrm{M}^{56}$. However, suppression of protease kinetics was identified at EGCG doses higher than $10 \mu \mathrm{M}$. Moreover, inhibition of virus production by THP-1 cells chronically infected with HIV-1 was promoted by liposome modification of EGCG in a dose-dependent manner. Following EGCG treatment of THP-1 cells inhibition of viral mRNA production was observed in lipopolysaccharide (LPS)activated chronically HIV-1-infected cells; however, inhibition of viral mRNA production in unstimulated or LPS-stimulated T-lymphoid cells (H9) was not observed $^{56}$. Another in vitro study showed that EGCG strongly inhibited the replication of HIV as determined by RT and p24 assays performed on cell supernatants ${ }^{55}$. Among catechin derivatives including ECG, EGC, EGCG and Green Tea Extract (GTE) with HIV-1 RT inhibitory activity, EGCG and ECG were recognized as highly potent HIV-1 RT inhibitors ${ }^{58}$. In addition, reduction by EGCG of HIV neurotoxicity in the presence of IFN- $\gamma$ via inhibition of Janus Activating Kinase/Signal Transducer and Activator of Transcription (JAK/STAT) pathway has been demonstrated in vivo ${ }^{64}$.

\subsection{Pu-erh Tea Polyphenols}

$\mathrm{Pu}$-erh tea is a dark, aged tea that is produced in Yunnan province, China, from a large-leaved variety of tea plant, Camellia sinensis assamica, following a two-step process of microbial fermentation by organisms such as the mould Aspergillus sp. and oxidation ${ }^{65,66}$. Several beneficial activities of $\mathrm{Pu}$-erh tea are documented, including prevention and treatment of cancer, heart diseases, rheumatoid arthritis and immune disease such as AIDS ${ }^{67-69}$. Due to its inhibitory effect on HIV replication $\mathrm{Pu}$-erh tea extracts may be used as a dietary supplement or a natural product administered to HIV patients ${ }^{65}$. Water and ethanol extracts of $\mathrm{Pu}$-erh tea (WEPT and EEPT), as well as fermented Pu-erh tea (FPT), possess anti-HIV activity with low cytotoxicity. The WEPT showed better anti-HIV activity than EEPT. Furthermore, this activity of the FPT's water extracts was better than that of the untreated $\mathrm{Pu}$-erh tea. As shown in Table 1, it has been reported that HIV- $1_{\mathrm{RF}}$ and HIV-2 ${ }_{\mathrm{CBL}-20}$ infections can be inhibited by Pu-erh tea extracts. Pu-erh tea reduced p24 antigen expression in HIV-1 $1_{\text {IIIB }}$ acutely infected C8166 cells and HIV$1_{\mathrm{KM} 018}$ infected peripheral blood mononuclear cells. The fusion between normal C8166 cells and HIV-1 chronically infected $\mathrm{H} 9$ cells was blocked by $\mathrm{Pu}$-erh tea. The synergistic anti-HIV activity of Pu-erh tea in combination with the antiretroviral medication azidothymidine (AZT) was noted although no inhibitory effects on RT activity were observed ${ }^{65}$.

\subsection{Black Tea Polyphenols}

Chinese black tea is made from the small-leaved Camellia sinensis sinensis and is more oxidized and stronger in flavour compared to other varieties of tea. Theaflavins are polyphenols that are found naturally in black tea and which constitute approximately $2 \%$ of its dried water extract. Anti-bacterial, anti-viral, anti-inflammatory, anti-oxidative and anti-tumour activities have been 


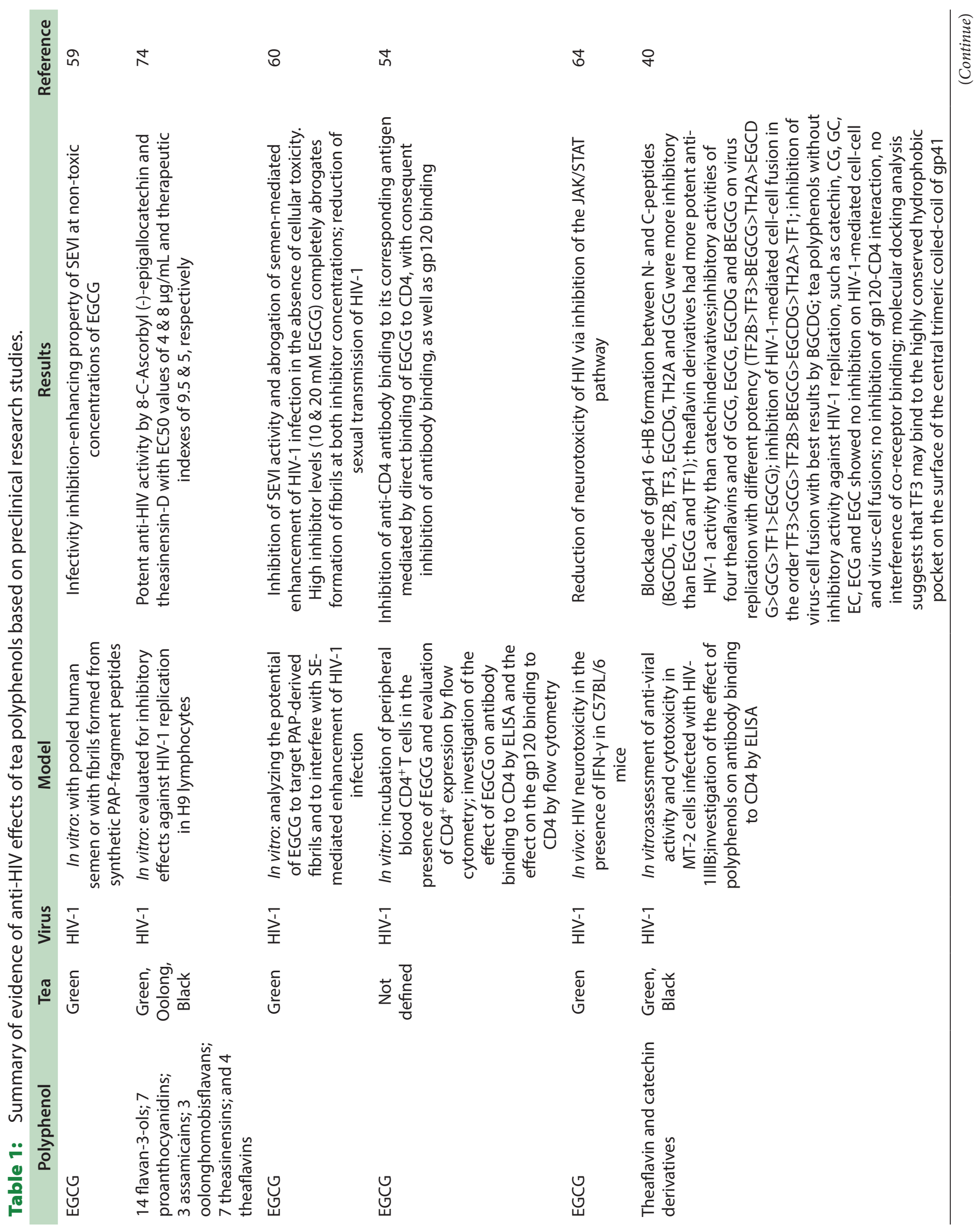




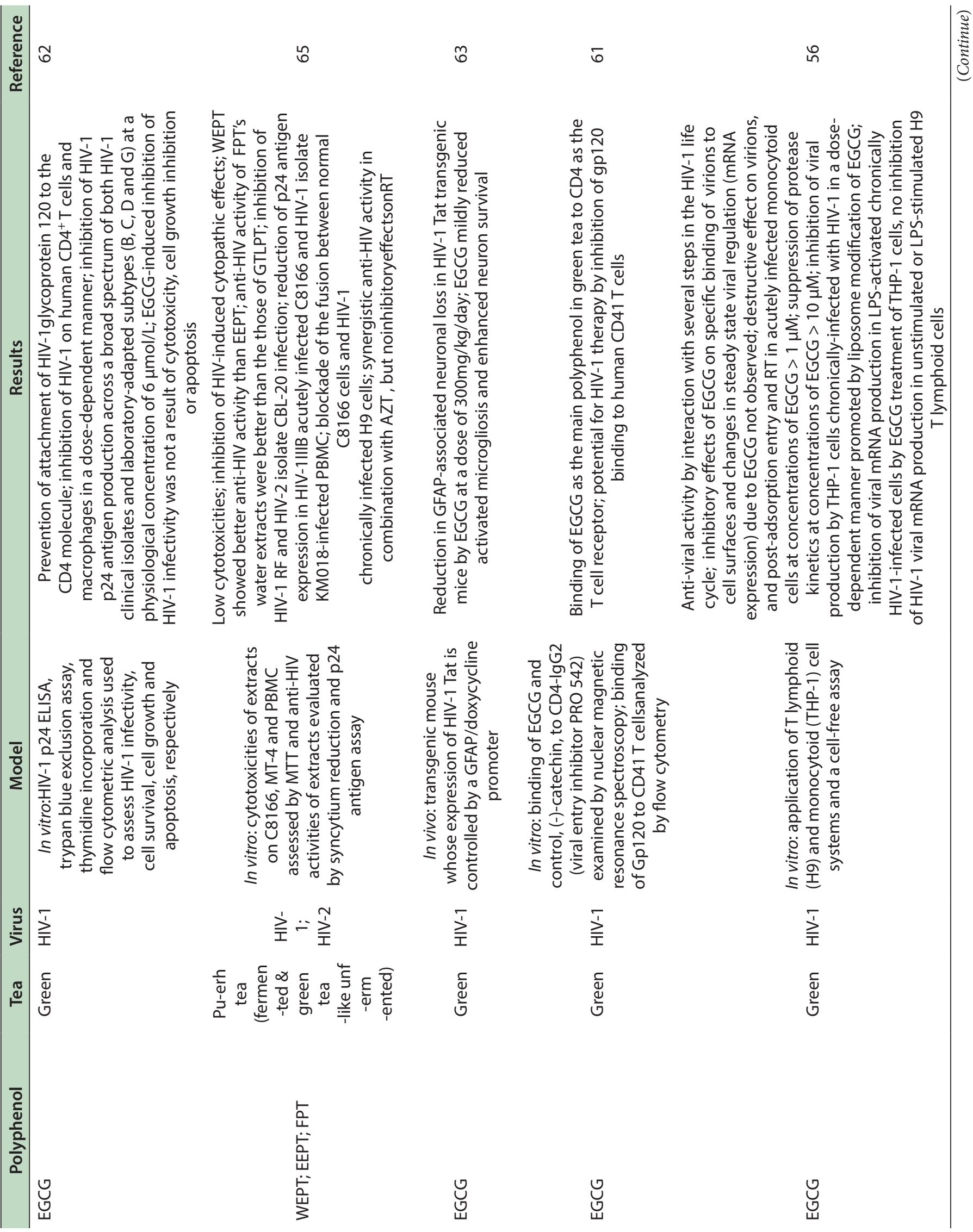




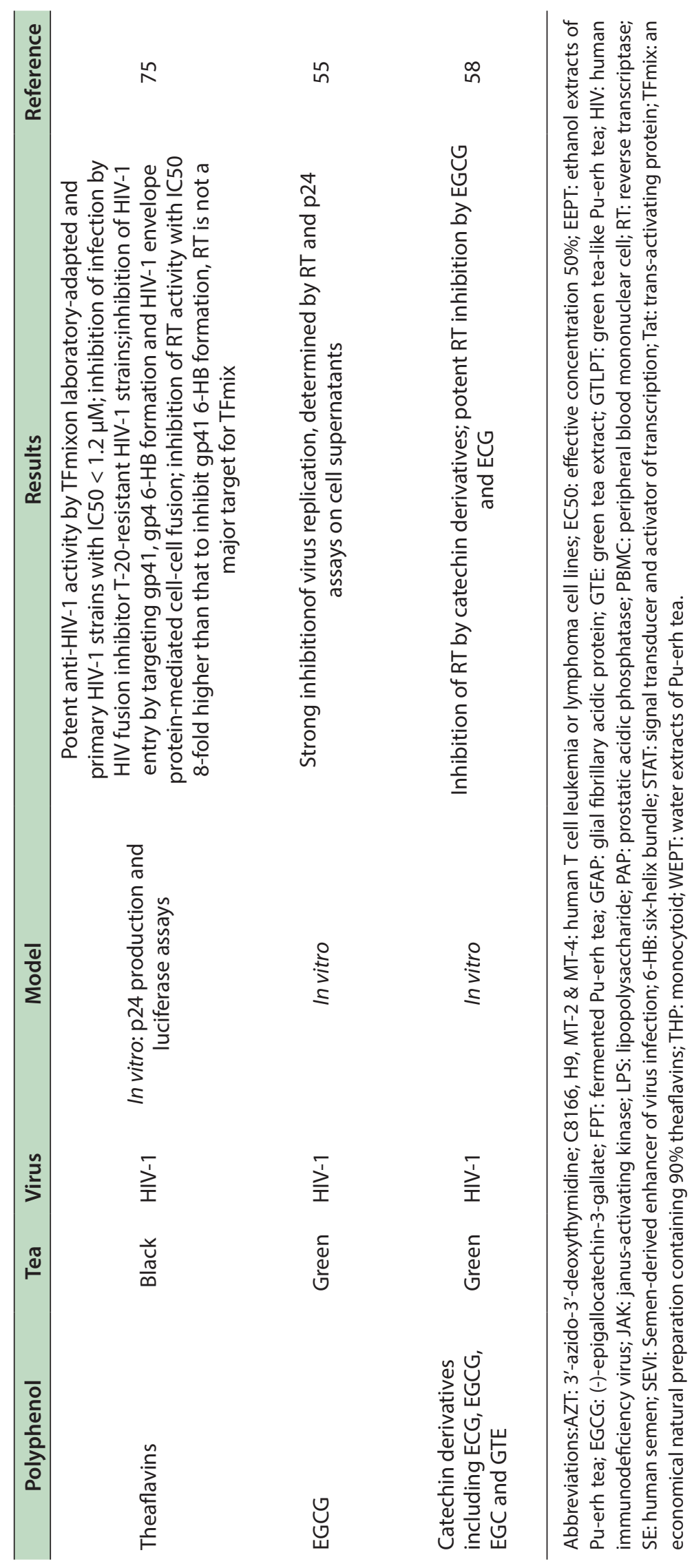


reported as properties of major theaflavin derivatives in black tea including theaflavin (TF1), theaflavin-3-gallate (TF2A), theaflavin-3'-gallate (TF2B) and theaflavin-3,3'digallate (TF3) ${ }^{70-73}$. It is known that theaflavins in black tea possess anti-HIV-1 activity through inhibition of virus entry into target cells by interfering with glycoprotein 41 six-helix bundle formation ${ }^{40}$. Due to higher antiHIV activity of black tea theaflavins in comparison to green tea catechins, extracts of the former are leading candidates for a new generation of anti-HIV-1 herbal therapy $^{74}$. Theaflavins and catechins both inhibit HIV-1 entry by targeting glycoprotein 41 between the $\mathrm{N}$ - and $\mathrm{C}$-peptides ${ }^{40}$. Amongst eight examined tea polyphenols, TF2B had the predominant anti-HIV activity followed in decreasing order by TF3, BEGCG, TH2A, EGCDG, GCG, TF1 and EGCG. Furthermore, the most inhibitory effect on HIV-1-mediated cell-cell fusion has been attributed to TF3 followed by GCG, TF2B, BEGCG, EGCDG, TH2A, TF1 and, poorly, EGCG. In contrast, neither inhibition of HIV-1-mediated cell-cell and virus-cell fusions, nor inhibitory activity against HIV1 replication have been observed for some other tea polyphenols including (-)-catechin, CG, GC, EC, ECG and $\mathrm{EGC}^{40}$.

It is thought that the mechanism of anti-HIV activity of tea polyphenols is not via specific interaction or interference with co-receptor binding. According to molecular docking analysis TF3 may bind to a highly conserved hydrophobic pocket that is located on the surface of the central trimeric coiled-coil of glycoprotein $41^{40}$. Another in vitro investigation using $\mathrm{p} 24$ production and luciferase assays demonstrated that a natural preparation containing $90 \%$ theaflavins (TFmix) had a strongly anti-HIV-1 effect on both laboratory-adapted and primary HIV-1 strains possessing low cytotoxicity ${ }^{75}$. Inhibition occurred at viral entry of the host cell by targeting glycoprotein 41 and blocking membrane fusion. While RT activity of the virus is also suppressed by TFmix, the $\mathrm{IC}_{50}$ for this inhibition is approximately 8 -fold higher than that needed for inhibition of glycoprotein $416-\mathrm{HB}$ formation, thus indicating that RT is not a primary target for $\mathrm{TFmix}^{74}$. In an animal model of arthritis black tea also showed anti-inflammatory activity ${ }^{76}$, demonstrating the immunomodulatory properties of theaflavins, which may be considered as complementary to their apparent protective effect against HIV-1 discussed here.

\section{Discussion}

According to the World Health Organization, around 71 million persons have become infected with HIV since records began, of whom 34 million patients have died due to AIDS-related illnesses, with a further 36.9 million individuals identified as carrying HIV at the end of $2014^{77}$. Current medicaments for AIDS include nucleoside RT inhibitors, nucleotide RT inhibitor, protease inhibitors, fusion inhibitors, co-receptor inhibitors and integrase inhibitor ${ }^{17}$, but which in some patients may cause a range of adverse side effects like liver disorders, muscular dystrophy, metabolic disorders, blood disorders, hypersensitivity reactions, pancreatitis and mitochondrial toxicity ${ }^{1,18,19}$. Multi-drug resistance and toxicity linked to the high genetic variability of the virus are additional limitations of conventional HIV treatments that collectively have prompted renewed efforts to discover novel therapeutic options.

Since ancient times humans have used plant-derived agents as treatments for various infective diseases and other ailments. The wealth of botanical-based therapies that are both efficacious and safe has prompted research to screen different known medicinal plants for antiHIV activity, of which different types of tea - with their broad range of polyphenols as an active ingredient could be considered as among the most important ${ }^{41}$. Green, Pu-erh and black teas contain such phenolic compounds as ECG, EGC, EGCG, GCG and TF which have demonstrated anti-HIV activity in several in vitro and in vivo studies (Table 1). While both are classified as flavonoids, green tea polyphenols consist mostly of EGCG and other catechin gallates, whereas theaflavins are the main constituents of black tea.

These two main categories of tea polyphenols are involved in cellular pathways such as JAK/STAT ${ }^{64}$, as well as in differentsteps of cell-cell and virus-cell interactions ${ }^{40}$. They show anti-viral and immunomodulatory effects on HIV-induced infection via different mechanisms including inhibition of virus replication, of HIV-induced cytopathic activities, and of antigen and RT expression, prevention of virus attachment and destruction of virions. Tea polyphenols also show activities against HIV infection in concentrations that are non-toxic for eukaryotic cell lines ${ }^{61}$. Pu-erh tea has been studied mostly in the form of crude extracts and isolated compounds 


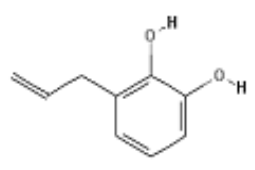

A

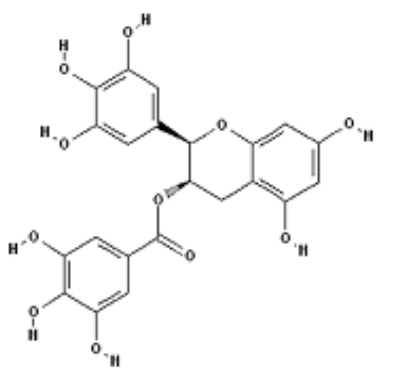

$\mathrm{E}$

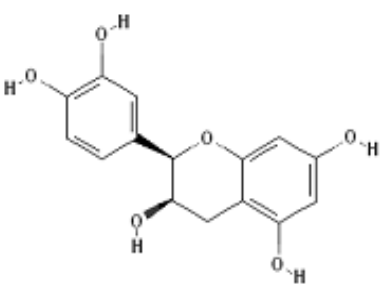

B

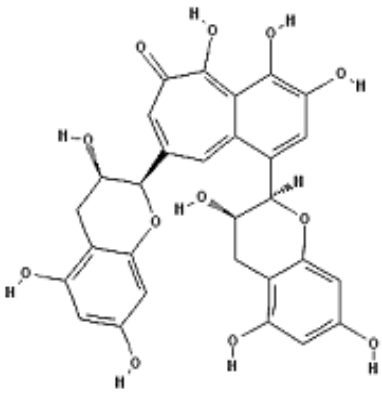

$\mathrm{F}$

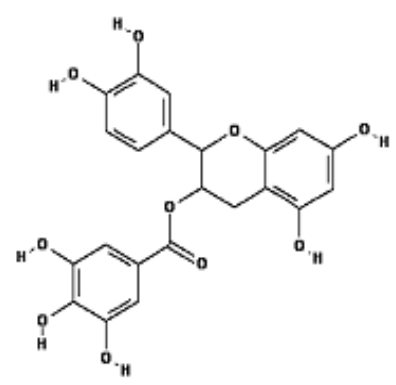

$\mathrm{C}$

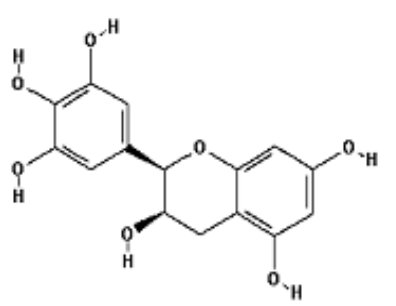

$\mathrm{D}$

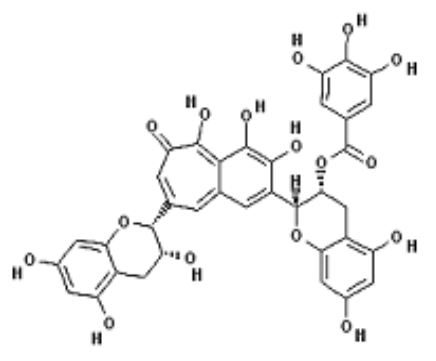

G

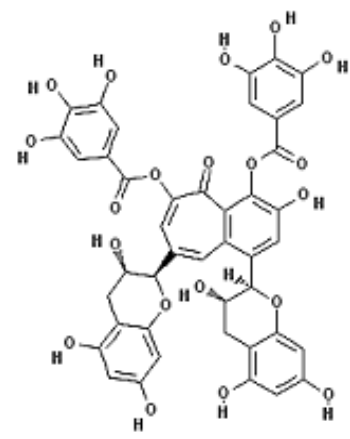

$\mathrm{H}$

Fig. 1. Chemical structure of tea polyphenols with major anti-HIV activity. A: catechin; B: (-)-epicatechin; C: (-)-epicatechingallate; D: (-)-epigallocatechin; E: (-)-epigallocatechingallate; F: theaflavin; G: theaflavin-3-gallate; $\mathrm{H}$ : theaflavin-3,3'-digallate.

were not assessed; however, these preparations were successful in demonstrating anti-HIV properties.

Figure 1 illustrates the chemical structure of each of the tea polyphenols with anti-HIV activity most relevant to future drug preparation. As one of the most significant properties of polyphenols, anti-oxidant effects are likely to play a crucial role in their noted antiretroviral activities. In addition, an immunomodulatory effect could be one of the mechanisms involved in the anti-HIV function of these molecules.

\section{Conclusion}

Taking into account the collective body of research discussed herein, it is proposed that tea polyphenols and polyphenol-rich extracts could be used in future as dietary supplement in combination with conventional anti-HIV medicines. Phenolic structures may also be considered as scaffolds for the discovery of a new generation of anti-HIV drugs. Since investigations of the protective effects of specific tea polyphenols in humans are either limited or have not yet taken place, there is a pressing need to undertake detailed clinical trials in order to attain sufficient and robust data to either support or refute the predicted possible beneficial role of tea polyphenols in the therapy of HIV-infected patients.

\section{Conflict of Interests}

The authors do not have any conflict of interest to declare. This research received no specific grant from any funding agency in the public, commercial or notfor-profit sectors.

\section{References}

1. Ramana LN, Anand AR, Sethuraman S, Krishnan UM. Targeting strategies for delivery of anti-HIV drugs. J Control Release. 2014; 192:271-83.

2. Sharp PM, Hahn BH. Origins of HIV and the AIDS pandemic. Cold Spring Harb Perspect Med. 2011; 1:a006841.

3. Sharp PM, Bailes E, Chaudhuri RR, Rodenburg CM, Santiago MO, Hahn BH. The origins of acquired immune 
deficiency syndrome viruses: where and when? Philos Trans R Soc Lond B Biol Sci. 2001; 356:867-76.

4. Maartens G, Celum C, Lewin SR. HIV infection: epidemiology, pathogenesis, treatment, and prevention. Lancet. 2014; 384:258-71.

5. Aldrich C, Hemelaar J. Global HIV-1 diversity surveillance. Trends Mol Med. 2012; 18:691-4.

6. Hemelaar J, Gouws E, Ghys PD, Osmanov S. WHOUNAIDS Network for HIV Isolation and Characterization. Global trends in molecular epidemiology of HIV-1 during 2000-2007. AIDS 2011; 25:679-89.

7. Crawford ND, Vlahov D. Progress in HIV reduction and prevention among injection and non-injection drug users. J Acquir Immune Defic Syndr. 2010; 55(S2):S84-7.

8. Donovan S. Pediatric HIV: current recommendations for diagnosis and treatment. Clin Microbiol Newsl. 2013; 35:127-31.

9. Duliege AM, Messiah A, Blanche S, Tardieu M, Griscelli C, Spira A. Natural history of human immunodeficiency virus type 1 infection in children: prognostic value of laboratory tests on the bimodal progression of the disease. Pediatr Infect Dis J. 1992; 11:630-5.

10. Reitz MS, Gallo RC. Human immunodeficiency virus. In: Mandell GL, Bennett JE, Dolin R (eds.) Mandell, Douglas and Bennett's Principles and Practice of Infectious Diseases, $7^{\text {th }}$ ed. Philadelphia: Churchill Livingstone; 2010. p. 2323-33.

11. Spira R, Lepage P, Msellati P, Van De Perre P, Leroy $\mathrm{V}$, Simonon A, et al. Natural history of human immunodeficiency virus type 1 infection in children: a five-year prospective study in Rwanda, Mother-to-Child HIV-1 Transmission Study Group. Pediatrics. 1999; 104:e56.

12. UNAIDS. AIDS by the numbers 2015. Geneva: 2015. Available from http://www.unaids.org/sites/default/ files/media_asset/AIDS_by_the_numbers_2015_en.pdf [Accessed on 13 May 2016].

13. Sabapathy K. Global roll-out of HIV treatment. Medicine. 2013; 41:479-82.

14. Kenya National Bureau of Statistics. Kenya Demographic and Health Survey, 2008-09. Calverton, Maryland: CBS \& ICF Macro; 2010. Available from http://dhsprogram.com/ pubs/pdf/fr229/fr229.pdf [Accessed on 13 May 2016].

15. Centers for Disease Control and Prevention. Estimated HIV incidence in the United States, 20072010. HIV Surveillance Supplemental Report. 2012; 17(4). Available from http://www.cdc.gov/hiv/pdf/ statistics_hssr_vol_17_no_4.pdf [Accessed on 13 May 2016].

16. National Health and Family Planning Commission of the People's Republic of China. 2015 China AIDS Response Progress Report. Geneva: 2015. Available from http:// www.unaids.org/sites/default/files/country/documents/ CHN_narrative_report_2015.pdf [Accessed on 13 May 2016].

17. De Clercq E. The history of antiretrovirals: key discoveries over the past 25 years. Rev Med Virol. 2009; 19:287-99.

18. Narayan LC, Rai VR, Tewtrakul S. Emerging need to use phytopharmaceuticals in the treatment of HIV. J Pharm Res. 2013; 6:218-23.

19. Panel on Antiretroviral Guidelines for Adults and Adolescents. Guidelines for the Use of Antiretroviral Agents in HIV-1-Infected Adults and Adolescents. US Department of Health and Human Services, 2016. Available from https://aidsinfo.nih.gov/contentfiles/ lvguidelines/adultandadolescentgl.pdf [Accessed on 13 May 2016].

20. Wainberg MA, Friedland G. Public health implications of antiretroviral therapy and HIV drug resistance. JAMA. 1998; 279:1977-83.

21. Ferrazzano GF, Roberto L, Amato I, Cantile T, Sangianantoni G, Ingenito A. Antimicrobial properties of green tea extract against cariogenic microflora: an in vivo study. J Med Food 2011; 14:907-11.

22. Farzaei MH, Rahimi R, Abdollahi M. The role of dietary polyphenols in the management of inflammatory bowel disease. Curr Pharm Biotechnol. 2015; 16:196-210.

23. Sodagari HR, Farzaei MH, Bahramsoltani R, Abdolghaffari AH, Mahmoudi M, Rezaei N. Dietary anthocyanins as a complementary medicinal approach for management of inflammatory bowel disease. Expert Rev Gastroenterol Hepatol. 2015; 9:807-20.

24. Farzaei MH, Farzaei F, Gooshe M, Abbasabadi Z, Rezaei $\mathrm{N}$, Abdolghaffari AH. Potentially effective natural drugs in treatment for the most common rheumatic disorder: osteoarthritis. Rheumatol Int. 2015; 35:799-814.

25. Scalbert A, Manach C, Morand C, Rémésy C, Jiménez L. Dietary polyphenols and the prevention of diseases. Crit Rev Food Sci Nutr. 2005; 45:287-306.

26. Khan N, Mukhtar H. Tea polyphenols for health promotion. Life Sci. 2007; 81:519-33.

27. UK Tea and Infusions Association. Tea Glossary and FAQ's. Available from https://www.tea.co.uk/tea-faqs [Accessed on 13 May 2016]. 
28. Food and Agriculture Organization of the United Nations Statistics Division - Production. Rome: FAOSTAT, 2015. Available from http://faostat3.fao.org/browse/Q/QC/E [Accessed on 13 May 2016].

29. Wierzejska R. Tea and health - a review of the current state of knowledge. Przegl Epidemiol. 2014; 68:501-6, 595-9.

30. EFSA Panel on Dietetic Products, Nutrition and Allergies. Scientific Opinion on the substantiation of health claims related to Camellia sinensis (L.) Kuntze (tea), including catechins in green tea and tannins in black tea, and protection of DNA, proteins and lipids from oxidative damage(ID 1103, 1276, 1311, 1708, 2664), reduction of acid production in dental plaque (ID 1105, 1111), maintenance of bone (ID 1109), decreasing potentially pathogenic intestinal microorganisms (ID 1116), maintenance of vision (ID 1280), maintenance of normal blood pressure (ID 1546) and maintenance of normal blood cholesterol concentrations (ID 1113, 1114) pursuant to Article 13(1) of Regulation (EC) No 1924/2006. EFSA Journal 2010; 8:1463.

31. Otsuka T, Ogo T, Eto T, Asano Y, Suganuma M, Niho Y. Growth inhibition of leukemic cells by (-) epigallocatechin gallate, the main constituent of green tea. Life Sci. 1998; 63:1397-403.

32. Baliga MS, Meleth S, Katiyar SK. Growth inhibitory and antimetastatic effect of green tea polyphenols on metastasis-specific mouse mammary carcinoma 4T1 cells in vitro and in vivo systems. Clin Cancer Res. 2005; 11:1918-27.

33. Shoji Y, Nakashima H. Glucose-lowering effect of powder formulation of African black tea extract in KK-A(y)/TaJcl diabetic mouse. Arch Pharm Res. 2006; 29:786-94.

34. Erba D, Riso P, Bordoni A, Foti P, Biagi PL, Testolin G. Effectiveness of moderate green tea consumption on antioxidative status and plasma lipid profile in humans. J Nutr Biochem. 2005; 16:144-9.

35. Mukamal KJ, Maclure M, Muller JE, Sherwood JB, Mittleman MA. Tea consumption and mortality after acute myocardial infarction. Circulation 2002; 105:247681.

36. Ramassamy C. Emerging role of polyphenolic compounds in the treatment of neurodegenerative diseases: a review of their intracellular targets. Eur J Pharmacol. 2006; 545:5164.

37. Shimamura T, Zhao W-H, Hu Z-Q. Mechanism of action and potential for use of tea catechin as an antiinfective agent. Anti-Infect Agents Med Chem. 2007; 6:57-62.
38. Park BJ, Park J-C, Taguchi H, Fukushima K, Hyon S-H, Takatori K. Antifungal susceptibility of epigallocatechin 3-O-gallate (EGCg) on clinical isolates of pathogenic yeasts. Biochem Biophys Res Commun. 2006; 347:4015.

39. Huang S-H, Tang YZ, Zhou X-M, Xie G, Kurihara H, He Z, et al. Study on anti-influenza virus effect of tea polyphenols in vitro and in vivo. Chaye Kexue. 2010; 30:302-8.

40. Liu S, Lu H, Zhao Q, He Y, Niu J, Debnath AK, et al. Theaflavin derivatives in black tea and catechin derivatives in green tea inhibit HIV-1 entry by targeting gp41. Biochim Biophys Acta. 2005; 1723:270-81.

41. Kanwar J, Taskeen M, Mohammad I, Huo C, Chan TH, Dou QP. Recent advances on tea polyphenols. Front Biosci. 2012; 4:111-31.

42. Morel I, Lescoat G, Cogrel P, Sergent O, Pasdeloup N, Brissot $\mathrm{P}$, et al. Antioxidant and iron-chelating activities of the flavonoids catechin, quercetin and diosmetin on ironloaded rat hepatocyte cultures. Biochem Pharmacol. 1993; 45:13-19.

43. Kono K, Tatara I, TakedaS, Arakawa K, Hara Y. Antibacterial activity of epigallocatechin gallate against methicillinresistant Staphylococcus aureus. Kansenshogaku Zasshi. $1994 ; 68: 1518-22$.

44. Amarowicz R, Pegg RB, Bautista DA. Antibacterial activity of green tea polyphenols against Escherichia coli K12. Nahrung. 2000; 44:60-2.

45. Yang CS. Inhibition of carcinogenesis by tea. Nature. 1997; 389:134-5.

46. Farzaei MH, Abdollahi M, Rahimi R. Role of dietary polyphenols in the management of peptic ulcer. World J Gastroenterol. 2015; 21:6499-517.

47. Maeda-Yamamoto M, Kawahara H, Tahara N, Tsuji K, Hara Y, Isemura M. Effects of tea polyphenols on the invasion and matrix metalloproteinases activities of human fibrosarcoma HT1080 cells. J Agric Food Chem. 1999; 47:2350-4.

48. NihalM,AhmadN,MukhtarH,WoodGS.Anti-proliferative and proapoptotic effects of (-)-epigallocatechin-3gallate on human melanoma: possible implications for the chemoprevention of melanoma. Int J Cancer. 2005; 114:513-21.

49. Frei B, Higdon JV. Antioxidant activity of tea polyphenols in vivo: evidence from animal studies. J Nutr. 2003; 133:3275S-84S.

50. Kim S-H, Jun C-D, Suk K, Choi B-J, Lim H, Park $\mathrm{S}$, et al. Gallic acid inhibits histamine release and 
pro-inflammatory cytokine production in mast cells. Toxicol Sci. 2006; 91:123-31.

51. Saleh F, Raghupathy R, Asfar S, Oteifa M, Al-Saleh N. Analysis of the effect of the active compound of green tea (EGCG) on the proliferation of peripheral blood mononuclear cells. BMC Complement Altern Med. 2014; 14:322.

52. Molina N, Bolin AP, Otton R. Green tea polyphenols change the profile of inflammatory cytokine release from lymphocytes of obese and lean rats and protect against oxidative damage. Int Immunopharmacol. 2015; 28:98596.

53. Marinovic MP, Morandi AC, Otton R. Green tea catechins alone or in combination alter functional parameters of human neutrophils via suppressing the activation of TLR-4/NFkB p65 signal pathway. Toxicol In Vitro. 2015; 29:1766-78.

54. Kawai K, Tsuno NH, Kitayama J, Okaji Y, Yazawa K, Asakage $\mathrm{M}$, et al. Epigallocatechin gallate, the main component of tea polyphenol, binds to CD4 and interferes with gp120 binding. J Allergy Clin Immunol. 2003; 112:951-7.

55. Fassina G, Buffa A, Benelli R, Varnier OE, Noonan DM, Albini A. Polyphenolic antioxidant (-)-epigallocatechin3-gallate from green tea as a candidate anti-HIV agent. AIDS 2002; 16:939-41.

56. Yamaguchi K, Honda M, Ikigai H, Hara Y, Shimamura T. Inhibitory effects of (-)-epigallocatechin gallate on the life cycle of human immunodeficiency virus type 1 (HIV-1). Antiviral Res. 2002; 53:19-34.

57. Haneda E, Furuya T, Asai S, Morikawa Y, Ohtsuki K. Biochemical characterization of casein kinase II as a protein kinase responsible for stimulation of HIV-1 protease in vitro. Biochem Biophys Res Commun. 2000; 275:434-9.

58. Tao P. The inhibitory effects of catechin derivatives on the activities of human immunodeficiency virus reverse transcriptase and DNA polymerases. Zhongguo Yi Xue Ke Xue Yuan Xue Bao. 1992; 14:334-8.

59. Hartjen P, Frerk S, Hauber I, Matzat V, Thomssen A, Holstermann B, et al. Assessment of the range of the HIV1 infectivity enhancing effect of individual human semen specimen and the range of inhibition by EGCG. AIDS Res Ther. 2012; 9:2.

60. Hauber I, Hohenberg H, Holstermann B, Hunstein W, Hauber J. The main green tea polyphenol epigallocatechin3-gallate counteracts semen-mediated enhancement of HIV infection. Proc Natl Acad Sci USA 2009; 106:9033-8.
61. Williamson MP, McCormick TG, Nance CL, Shearer WT. Epigallocatechin gallate, the main polyphenol in green tea, binds to the T-cell receptor, CD4: potential for HIV-1 therapy. J Allergy Clin Immunol. 2006; 118:1369-74.

62. Nance CL, Siwak EB, Shearer WT. Preclinical development of the green tea catechin, epigallocatechin gallate, as an HIV-1 therapy. J Allergy Clin Immunol. 2009; 123:45965.

63. Rrapo E, Zhu Y, Tian J, Hou H, Smith A, Fernandez F, et al. Green tea-EGCG reduces GFAP associated neuronal loss in HIV-1 Tat transgenic mice. Am J Transl Res. 2009; 1:72-9.

64. Giunta B, Obregon D, Hou H, Zeng J, Sun N, Nikolic V, et al. EGCG mitigates neurotoxicity mediated by HIV-1 proteins gp120 and Tat in the presence of IFN- $\gamma$ : role of JAK/STAT1 signaling and implications for HIV-associated dementia. Brain Res. 2006; 1123:216-25.

65. Huang N, Yang L-M, Li X-L, Zheng C-B, Wang R-R, Yang $\mathrm{Y}-\mathrm{P}$, et al. Anti-HIV activities of extracts from Pu-erh tea. Chin J Nat Med. 2012; 10:347-52.

66. Kubota K, Sumi SH, Tojo H, Sumi-Inoue Y, I-chin H, Oi $\mathrm{Y}$, et al. Improvements of mean body mass index and body weight in preobese and overweight Japanese adults with black Chinese tea (Pu-Erh) water extract. Nutr Res. 2011; 31:421-8.

67. Duh P-D, Yen G-C, Yen W-J, Wang B-S, Chang L-W. Effects of pu-erh tea on oxidative damage and nitric oxide scavenging. J Agric Food Chem. 2004; 52:8169-76.

68. Kuo K-L, Weng M-S, Chiang C-T, Tsai Y-J, Lin-Shiau S-Y, Lin J-K. Comparative studies on the hypolipidemic and growth suppressive effects of oolong, black, pu-erh, and green tea leaves in rats. J Agric Food Chem. 2005; 53:4809.

69. Way T-D, Lin H-Y, Kuo D-H, Tsai S-J, Shieh J-C, Wu JC, et al. $\mathrm{Pu}$-erh tea attenuates hyperlipogenesis and induces hepatoma cells growth arrest through activating AMPactivated protein kinase (AMPK) in human HepG2 cells. J Agric Food Chem. 2009; 57:5257-64.

70. Yang CS, Landau JM. Effects of tea consumption on nutrition and health. J Nutr. 2000; 130:2409-12.

71. Higdon JV, Frei B. Tea catechins and polyphenols: health effects, metabolism, and antioxidant functions. Crit Rev Food Sci Nutr. 2003; 43:89-143.

72. Lambert JD, Yang CS. Mechanisms of cancer prevention by tea constituents. J Nutr. 2003; 133:3262S-7S.

73. Mukhtar H, Ahmad N. Tea polyphenols: prevention of cancer and optimizing health. Am J Clin Nutr. 2000; 71(S6):1698S-702S. 
74. Hashimoto F, Kashiwada Y, Nonaka G, Nishioka I, Nohara T, Cosentino LM, et al. Evaluation of tea polyphenols as anti-HIV agents. Bioorg Med Chem Lett. 1996; 6:695700.

75. Yang J, Li L, Tan S, Jin H, Qiu J, Mao Q, et al. A natural theaflavins preparation inhibits HIV-1 infection by targeting the entry step: potential applications for preventing HIV-1 infection. Fitoterapia. 2012; 83:348-55.
76. Chattopadhyay C, Chakrabarti N, Chatterjee M, Mukherjee S, Sarkar K, Chaudhuri AR. Black tea (Camellia sinensis) decoction shows immunomodulatory properties on an experimental animal model and in human peripheral mononuclear cells. Pharmacognosy Res. 2012; 4:15-21.

77. World Health Organization. Global Health Observatory (GHO) data. Geneva: WHO; 2016. Available from http:// www.who.int/gho/hiv/en/ [Accessed on 13 May 2016]. 